Real Analysis Exchange

Vol. 23(2), 1997-1998, pp. 709-717

Irene Mulvey, Fairfield University Department of Mathematics \& Computer

Science Fairfield, CT 06430-7524. e-mail: mulvey@fair1.fairfield.edu

\title{
A GEOMETRIC ALGORITHM TO DECIDE THE FORCING RELATION ON CYCLES
}

\begin{abstract}
The forcing relation was shown to be a partial order by S. Baldwin. Here we give a new, natural, geometric algorithm to determine if one cycle forces another.
\end{abstract}

\section{Introduction.}

In 1964, Sarkovskii [1] defined this linear order on the set of natural numbers. Sarkovskii order:

$$
\begin{gathered}
3,5,7,9, \cdots, 2 \cdot 3,2 \cdot 5,2 \cdot 7, \cdots, 2^{2} \cdot 3,2^{2} \cdot 5,2^{2} \cdot 7, \cdots, 2^{n} \cdot 3,2^{n} \cdot 5,2^{n} \cdot 7, \cdots, \\
\cdots, \cdots, \cdots, 2^{4}, 2^{3}, 2^{2}, 2,1
\end{gathered}
$$

He proved the following theorem.

Sarkovskii's Theorem.

Let $f: R \rightarrow R$ be a continuous map. If $f$ has a periodic point of least period $n$, then $f$ has a periodic point of least period $m$ for every $m$ to the right of $n$ in the Sarkovskii order.

In 1987, S. Baldwin [2] considered not only the least period of a periodic point but also the orbit type. He defined the forcing relation on finite cyclic permutations (cycles), proved that this relation is a partial order, and provided an exhaustive but inefficient algorithm to decide if one cycle forces another. Many people have continued the study of this forcing relation on cycles; see [3] sections $2.3-2.7$ for a thorough discussion. Of particular interest is the question of how to efficiently determine if one cycle forces another cycle (see [3] text following corollary 2.7.2). Using kneading theory and combinatorial arguments, Jungreis [4] provided an algorithm to determine if one cycle forces another. In the case when the two cycles have approximately the same number

Key Words: cycles and forcing relation

Mathematical Reviews subject classification: Primary: 58F03 Secondary: 58F20

Received by the editors October 15, 1996 
of critical points, he provides a polynomial-time algorithm to decide the forcing relation. In [5], a geometric proof of Jungreis' algorithm is given and in [6] this algorithm is generalized to two arbitrary cycles.

In this paper, we present (in section 5) a new, natural, geometric algorithm to determine if a cycle $\theta$ forces a cycle $\eta$. We use oriented graph machinery for $\theta$ (section 3) and we define a template for $\eta$ (section 4). The oriented graph used here is a bit more complicated than that described in [2] and [3], but will provide more information. This oriented graph enables one to rule out certain cycles as being forced by the given cycle. In particular, our oriented graph indicates the maximum number of consecutive moves to the right (and left) that a cycle forced by a given cycle can make. The idea of the template for a cycle is new. In section 6 , we give examples and remarks on how this outlook can be used to illustrate some of the structure of the total order induced by the forcing relation on unimodal cycles.

\section{Preliminaries}

Let $f: I \rightarrow I$ be a continuous map on a compact interval. Define $f^{0}(x)=x$ and for $n \in \mathbb{N}, f^{n}(x)=f\left(f^{n-1}(x)\right)$. For $x \in I$, the orbit of $x$ is the set

$$
\operatorname{orb}_{f}(x)=\left\{f^{i}(x): i=0,1,2, \cdots\right\}
$$

If there exists $k \in \mathbb{N}$ satisfying $f^{k}(x)=x$, we say $x$ is periodic for $f$ and $x$ has least period $s$ where $s$ is smallest in $\mathbb{N}$ such that $f^{s}(x)=x$. A point of least period 1 is called a fixed point for $f$. If $x$ is periodic for $f$ with least period $s$, then the orbit of $x$ is the finite set $\operatorname{orb}_{f}(x)=\left\{x, f(x), f^{2}(x), \cdots, f^{s-1}(x)\right\}$.

A cycle is a bijection $\eta:\{1,2, \cdots, m\} \rightarrow\{1,2, \cdots, m\}$ satisfying $\eta^{k}(1) \neq 1$ for $1 \leq k<m$. We will usually denote a cycle $\eta$ by $\eta=\left(k_{1}, k_{2}, \cdots, k_{m}\right)$ where $\eta\left(k_{i}\right)=k_{i+1}$ and $\eta\left(k_{m}\right)=k_{1}$. We will assume, without loss of generality, that $k_{1}=1$. Write the elements of a periodic orbit, $\operatorname{orb}_{f}(x)$, in increasing order: $x_{1}<x_{2}<\cdots<x_{s}$. We say $\operatorname{orb}_{f}(x)$ has orbit type $\eta$ if $\eta$ is a cycle on $\{1,2, \cdots, s\}$ and $f\left(x_{i}\right)=x_{\eta(i)}$. In fact, we will say any $x_{i}$ in $\operatorname{orb}_{f}(x)$ has orbit type $\eta$.

The forcing relation on cycles is defined as follows: $\theta$ forces $\eta$ if and only if every continuous map of the interval that has a periodic orbit of type $\theta$ has a periodic orbit of type $\eta$. Baldwin [2] proved that this relation induces a partial order on the set of cycles. In the rest of this paper, any periodic orbit is assumed to have period $s \geq 3$ since forcing on smaller periodic orbits and the corresponding cycles can be easily handled via special cases. Period doubles do not cause a problem either. A periodic orbit has an orbit type $\eta$ on $\{1,2, \cdots, m\}$ that is a period double if $m$ is even and $\eta$ cyclically permutes the 
sets $\{1,2\},\{3,4\}, \cdots,\{m-1, m\}$. And, $\eta$ is said to be a double of the cycle $\eta / 2$ on $\{1,2, \cdots, m / 2\}$ defined by $\eta / 2(i)=j$ if and only if $\eta(\{2 i-1,2 i\})=$ $\{2 j-1,2 j\}$. Every double forces the cycle it is a double of and no cycle can force a cycle $\theta$ without forcing every double of $\theta$ as well (see [7]). We may assume that we are not dealing with a period double since we can replace any period double $\eta$ with the cycle it is a double of.

\section{An Oriented Graph for $\theta$}

Let $\theta$ be a cycle on $\{1,2, \cdots, n\}$ with $n \geq 3$. In this section we define an oriented graph $M(\theta)$ for $\theta$ and obtain results concerning loops in $M(\theta)$. (The oriented graph constructed here is more complicated than the similar object in [2] and [3] in that it is constructed using the integers $\{1,2, \cdots, n\}$ and the fixed points of the $\theta$-linear map, but this more complicated construction yields more information. See [8] sections 1.1-1.4, [2] section 2, and [3] Chapter 1.

The $\theta$-linear map $f_{\theta}$ is the continuous function defined by $f_{\theta}:[1, n] \rightarrow[1, n]$ by $f_{\theta}=\theta$ on $\{1,2, \cdots, n\}$ and $f_{\theta}$ is linear on $[i, i+1]$ for $1 \leq i \leq n-1$. Let $P=\left\{p_{1}, p_{2}, \cdots, p_{k}\right\}$ denote the non-empty, finite set of fixed points of $f_{\theta}$. Since $P \cap\{1,2, \cdots, n\}=\emptyset$, we can list the elements of $P \cup\{1,2, \cdots, n\}$ in increasing order:

$$
1=q_{1}<q_{2}<\cdots<q_{n+k}=n
$$

These points determine a collection of $(n+k-1)$ non-degenerate, compact intervals with mutually disjoint interiors and union equal to $[1, n]$. We label each interval $L$ or $R$ depending on whether each element in the interior of the interval is mapped to its left or to its right by $f_{\theta}$ and we number the intervals in increasing order from left to right. Specifically, for $1 \leq i \leq n+k-1$,

$$
I_{i}=\left[q_{i}, q_{i+1}\right]= \begin{cases}L_{i} & \text { if } f_{\theta}(x)<x \text { for all } x \in\left(q_{i}, q_{i+1}\right) \\ R_{i} & \text { if } f_{\theta}(x)>x \text { for all } x \in\left(q_{i}, q_{i+1}\right) .\end{cases}
$$

Note that we always have $\left[1, q_{2}\right]=R_{1}$ and $\left[q_{n+k-1}, n\right]=L_{n+k-1}$. Let $G=\left\{I_{1}, I_{2}, \cdots, I_{n+k-1}\right\}$ denote the set of intervals and define a relation $R$ on $G$ as follows:

$$
I_{i} R I_{j} \Leftrightarrow f_{\theta}\left(I_{i}\right) \supseteq I_{j} .
$$

Define a function $\operatorname{sgn}: G \rightarrow\{+1,-1\}$ by

$$
\operatorname{sgn}\left(I_{i}\right)= \begin{cases}+1 & \text { if } f_{\theta} \text { has positive slope on } I_{i} \\ -1 & \text { if } f_{\theta} \text { has negative slope on } I_{i} .\end{cases}
$$


The oriented graph for $\theta$, denoted $M(\theta)$, is the triple $(G, R, s g n)$.

A finite path of length $s$ in $M(\theta)$ in an s-tuple $W=\left(I_{i_{1}}, I_{i_{2}}, \cdots, I_{i_{s}}\right) \in G^{S}$ such that $I_{i_{k}} R I_{i_{k+1}}$ for $1 \leq k \leq s-1$. If, in addition, $I_{i_{s}} R I_{i_{1}}$, we say the finite path is a loop of length s. A loop in $M(\theta)$ is repetitive if it is a shorter loop repeated more than once. That is, $W=\left(I_{i_{1}}, I_{i_{2}}, \cdots, I_{i_{s}}\right)$ is repetitive if there is a divisor $r$ of $s$ such that $I_{i_{j}}=I_{i_{j+r}}$ for $1 \leq j \leq s-r$. Otherwise, $W$ is non-repetitive. We state a slight variation of a well-known result. ([8] lemma 1.4, [3] lemma 1.2.7]).

Lemma 3.1. Let $\theta$ be a cycle with oriented graph $M(\theta)$. For each nonrepetitive loop, $W=\left(I_{i_{1}}, I_{i_{2}}, \cdots I_{i_{s}}\right)$, of length $s \geq 3$ in $M(\theta)$, there exists an $x$ in $[1, n]$ with least $f_{\theta}$-period $s$ and $f_{\theta}^{k}(x) \in I_{i_{k+1}}$ for $0 \leq k \leq s-1$.

In lemma 3.1, we must assume $s \geq 3$ because in our construction a nonrepetitive loop of length 2 may correspond to a fixed point. But, for nonrepetitive loops of length greater than 2 , there is no problem.

Since our oriented graph is signed, for any non-repetitive loop in $M(\theta)$, we can find the orbit type of the periodic point $x$ (as in [2] section 2, and [3] remark 2.6.7). The oriented graph in this paper is a bit more complicated, but the results are essentially the same. First, we define the orbit type of a non-repetitive loop in $M(\theta)$.

Define a strict linear order on distinct loops of the same length in $M(\theta)$ as follows. For $W=\left(I_{i_{1}}, I_{i_{2}}, \cdots, I_{i_{s}}\right)$ and $V=\left(I_{j_{1}}, I_{j_{2}}, \cdots, I_{j_{s}}\right)$,

$$
W<V \Leftrightarrow \prod_{n=1}^{m-1} \operatorname{sgn}\left(I_{i_{n}}\right) \cdot i_{m}<\prod_{n=1}^{m-1}\left(\operatorname{sgn}\left(I_{j_{n}}\right) \cdot j_{m}\right.
$$

where $m \leq s$ is smallest such that $i_{m} \neq j_{m}$. Define a natural shift operation on loops in $M(\theta)$ :

$$
\operatorname{sh}\left(I_{i_{1}}, I_{i_{2}}, \cdots, I_{i_{s}}\right)=\left(I_{i_{2}}, I_{i_{3}}, \cdots, I_{i_{s}}, I_{i_{1}}\right)
$$

If $W$ is a non-repetitive loop of length $s \geq 3$ in $M(\theta)$, let $\mathcal{W}$ be the smallest set of loops from $M(\theta)$ that contains $W$ and is closed under the shift operation. Let $g: \mathcal{W} \rightarrow\{1,2, \cdots s\}$ be the unique, order-preserving bijection and define a cycle $\pi_{W}$ by $\pi_{W}(i)=g \circ s h \circ g^{-1}(i)$ for $1 \leq i \leq s$. We define the orbit type of $W$ to be the cycle $\pi_{W}$. For $W=\left(I_{i_{1}}, I_{i_{2}}, \cdots I_{i_{s}}\right)$ a non-repetitive loop in $M(\theta)$, we will say that $x$ in $[1, n]$ has itinerary $W$ if $f_{\theta}^{k}(x) \in I_{i_{k+1}}$ for $0 \leq k \leq s-1$.

Lemma 3.2. Let $W$ and $V$ be distinct loops of the same length in $M(\theta)$. If $W<V$, then every $x$ with itinerary $W$ is less than every $y$ with itinerary $V$. 
Proof. Let $W=\left(I_{i_{1}}, I_{i_{2}}, \cdots, I_{i_{s}}\right)$ and $V=\left(I_{j_{1}}, I_{j_{2}}, \cdots, I_{j_{s}}\right)$. Say $m \leq s$ is smallest such that $i_{m} \neq j_{m}$. Since $W<V$, we must have either (1) $x$ and $y$ satisfy $f^{m-1}(x)<f^{m-1}(y)$ after having been mapped through an even number of orientation-reversing intervals, or (2) $x$ and $y$ satisfy $f^{m-1}(x)>f^{m-1}(y)$ after having been mapped through an odd number of orientation-reversing intervals. In either case, $x<y$.

Theorem 3.3. Let $\theta$ be a cycle with oriented graph $M(\theta)$. If $W$ is a nonrepetitive loop of length $s \geq 3$ in $M(\theta)$, then there exists a periodic point $x$ in $[1, n]$ with least $f_{\theta}$-period $s$ and the orbit type of $x$ is the same as the orbit type of $W$.

Proof. Say $W=\left(I_{i_{1}}, I_{i_{2}}, \cdots, I_{i_{s}}\right)$. By lemma 3.1 , there is $x$ in $[1, n]$ with least $f_{\theta}$-period $s$ and $f_{\theta}^{k}(x) \in I_{i_{k+1}}$ for $0 \leq k \leq s-1$. Write the orbit of $x$ in increasing order: $\operatorname{orb}_{f}(x)=\left\{x_{1}<x_{2}<\cdots x_{s}\right\}$. Write $\mathcal{W}$ in increasing order: $\mathcal{W}=\left\{W_{1}<W_{2}<\cdots<W_{s}\right\}$, where as before $\mathcal{W}$ is the smallest collection of loops that contains $W$ and is closed under the shift operation. Let $\pi_{W}$ be the cycle that denotes the orbit type of $W$ as previously defined. For each $i$, $x_{i}$ has itinerary $W_{i}$ by the order defined on walks. Let the orbit type of $x$ be given by the cycle $\varphi$. Now,

$$
\begin{aligned}
\pi_{W}(i) & =j \Leftrightarrow g \circ s h \circ g^{-1}(i)=j \\
& \Leftrightarrow s h \circ g^{-1}(i)=g^{-1}(j) \\
& \Leftrightarrow s h\left(W_{i}\right)=W_{j} \\
& \Leftrightarrow \text { the itinerary of } f\left(x_{i}\right)=\text { the itinerary of } x_{j} \\
& \Leftrightarrow f\left(x_{i}\right)=x_{j} \\
& \Leftrightarrow \varphi(i)=j
\end{aligned}
$$

\section{The Template for $\eta$.}

Let $\eta=\left(k_{1}, k_{2}, \cdots, k_{s}\right)$ be a cycle on $\{1,2, \cdots, s\}$ and assume, without loss of generality, that $k_{1}=1$. Define $p:\{1,2, \cdots, s\} \rightarrow\{1,2, \cdots, s\}$ by $p\left(k_{j}\right)=j$. That is ,

$$
p(m)=j \Leftrightarrow \eta^{j-1}(1)=m
$$

(So, $p$ describes the location of each integer in the orbit of 1.) We now define the template for $\eta$ which will be a collection of combinatorial data that captures the orbit structure of $\eta$. 
Definition. The template for $\eta$ is

(1) an $s$-tuple $I=\left(I_{1}, I_{2}, \cdots, I_{s}\right) \in\{R, L\}^{s}$

(2) an $(s-1)$-tuple $J=\left(m_{1}, m_{2}, \ldots, m_{s-1}\right) \in\{<, \leq\}^{s-1}$

(3) an $(s-1)$-tuple $K=\left(n_{1}, n_{2}, \cdots, n_{s-1}\right) \in\{0,+1,-1\}^{s-1}$

defined as follows. For $1 \leq j \leq s$ define

$$
I_{j}= \begin{cases}R & \text { if } \eta^{j}(1)>\eta^{j-1}(1) \\ L & \text { if } \eta^{j}(1)<\eta^{j-1}(1)\end{cases}
$$

And for $1 \leq j \leq s-1$ define

$$
\begin{gathered}
m_{j}= \begin{cases}< & \text { if } I_{p(j)} \neq I_{p(j+1)} \\
\leq & \text { if } I_{p(j)}=I_{p(j+1)}, \text { and }\end{cases} \\
n_{j}= \begin{cases}+1 & \text { if } m_{j} \text { is } \leq \text { and } \eta \text { is orientation-preserving on }\{j, j+1\} \\
-1 & \text { if } m_{j} \text { is } \leq \text { and } \eta \text { is orientation-reversing on }\{j, j+1\} \\
0 & \text { if } m_{j} \text { is }<.\end{cases}
\end{gathered}
$$

The definition of $n_{j}$ makes sense because $m_{j}$ is $\leq$ only when the two adjacent points $j$ and $j+1$ are both mapped to the right by $\eta$ or are both mapped to the left by $\eta$. Note that $I_{1}=R$ and $I_{s}=L$ always.

In practice, it is simple to write the template for $\eta$. (It is helpful to draw the orbit on a line.) The template for $\eta=(132)$ is $I=(R, L, L), J=(<$ , $\leq)$, and $K=(0,+1)$. The template for $\eta=(13542)$ is $I=(R, R, L, L, L)$, $J=(<,<,<, \leq)$, and $K=(0,0,0,+1)$. The template for $\eta=(1234)$ is $I=(R, R, R, L), J=(\leq, \leq,<)$, and $K=(+1,+1,0)$.

\section{Main theorem.}

In this section we prove the main theorem which provides a way to determine if one cycle $\theta$ forces another cycle $\eta$. Assume $\theta$ is a cycle with oriented graph $M(\theta)$ as defined in section 3 . Assume $\eta=\left(k_{1}, k_{2}, \cdots, k_{s}\right)$ is a cycle on $\{1,2, \cdots, s\}$ with $k_{1}=1, \eta$ has template $(I, J, K)$ given by

$$
\begin{aligned}
I & =\left(I_{1}, I_{2}, \cdots, I_{s}\right) \in\{R, L\}^{s} \\
J & =\left(m_{1}, m_{2}, \cdots, m_{s-1}\right) \in\{<, \leq\}^{s-1} \\
K & =\left(n_{1}, n_{2}, \cdots, n_{s-1}\right) \in\{+1,-1,0\}^{s-1}
\end{aligned}
$$


and as before, $p:\{1,2, \cdots, s\} \rightarrow\{1,2, \cdots, s\}$ is defined by $p\left(k_{j}\right)=j$.

Definition. A non-repetitive loop $W$ of length $s$ from $M(\theta)$

$$
W=\left(H_{i_{1}}, H_{i_{2}}, \cdots, H_{i_{s}}\right)
$$

fits the template for $\eta$ if and only if

(1) for all $1 \leq j \leq s, H_{i_{j}}=I_{j}$

(2) for all $1 \leq j \leq s-1, i_{p(j)} m_{j} i_{p(j+1)}$

(3) for all $1 \leq j \leq s-1$, if $i_{p(j)}=i_{p(j+1)}$, then $\operatorname{sgn}\left(H_{i_{p(j)}}\right)=n_{j}$

The idea is this: the data contained in the template of $\eta$ captures the orbit structure of $\eta$ so that any loop in $M(\theta)$ that fits the template of $\eta$ will guarantee the existence of a periodic point for $f_{\theta}$ that has orbit type $\eta$.

Lemma 5.1. Let $W=\left(H_{i_{1}}, H_{i_{2}}, \cdots, H_{i_{s}}\right)$ be a non-repetitive loop that fits the template for $\eta$, then for $1 \leq j \leq s-1$ the loop that is a shift of $W$ and begins with $H_{i_{p(j)}}$ is the $j$ th smallest walk in $\mathcal{W}$.

Proof. We will show that the loop beginning with $H_{i_{p(j)}}$ is smaller than the loop beginning with $H_{i_{p(j+1)}}$. In any event, since $W$ fits the template for $\eta$, we have $i_{p(j)} \leq i_{p(j+1)}$. If $i_{p(j)}<i_{p(j+1)}$, then the result immediately follows. If $i_{p(j)}=i_{p(j+1)}$, then $\operatorname{sgn}\left(H_{i_{p(j)}}\right)=n_{j}$ is either +1 or -1 . If $\operatorname{sgn}\left(H_{i_{p(j)}}\right)=+1$ , then $\eta$ is orientation-preserving on $\{j, j+1\}$, so $\eta(j)<\eta(j+1)$. Then, $i_{p(\eta(j))} \leq i_{p(\eta(j+1))}$. As before, if $i_{p(\eta(j))}<i_{p(\eta(j+1))}$, then the result follows. If $\operatorname{sgn}\left(H_{i_{p(j)}}\right)=-1$, then $\eta$ is orientation-reversing on $\{j, j+1\}$, so $\eta(j)>$ $\eta(j+1)$. Then, $i_{p(\eta(j))} \geq i_{p(\eta(j+1))}$. If $i_{p(\eta(j))}>i_{p(\eta(j+1))}$, then the result follows. The lemma follows by repeating this argument.

Lemma 5.2. If $W$ is a non-repetitive loop that fits the template of $\eta$, then the orbit type of $W$ is $\eta$.

Proof. Suppose $W$ has orbit type $\pi$. Then,

$$
\begin{aligned}
\pi(b) & =g \circ s h \circ g^{-1}(b) \\
& =g \circ s h\left[H_{i_{p(b)}}, H_{i_{p(b)+1}}, \cdots H_{i_{p(b)-1}}\right] \\
& =g\left[H_{i_{p(b)+1}}, H_{i_{p(b)+2}}, \cdots H_{i_{p(b)}}\right] \\
& =g\left[H_{i_{p(\eta(b))}}, H_{i_{p(\eta(b))+1}}, \cdots H_{i_{p(\eta(b))-1}}\right] \\
& =\eta(b)
\end{aligned}
$$

since $p(b)+1=p(\eta(b))$. 
Theorem 5.3. $\theta$ forces $\eta \Leftrightarrow \theta=\eta$ or there is a non-repetitive loop in $M(\theta)$ that fits the template for $\eta$

Proof. [ $\Leftarrow]$ We have a loop $W$ in $M(\theta)$ that fits the template for $\eta$. By theorem 3.3, there is $x \in[1, n]$ with least $f_{\theta}$-period $s$ and the orbit type of $x$ is the orbit type of the $W$. By lemma 5.2, $x$ has orbit type $\eta$.

$[\Rightarrow] \theta$ forces $\eta$ and $\theta \neq \eta$, so $f_{\theta}$ has a periodic point $x$ with orbit type $\eta$. Then, $W$ defined by $W=\left(I_{i_{1}}, I_{i_{2}}, \cdots, I_{i_{s}}\right)$ where $f^{j-1}(x) \in I_{i_{j}}$ for $1 \leq j \leq s$ is a non-repetitive loop in $M(\theta)$ that fits the template for $\eta$.

\section{Examples and Remarks.}

Let $\theta=(1234)$. The oriented graph for $\theta$ is given by $M(\theta)$ :

$$
\begin{aligned}
& R_{1}(+) \rightarrow R_{2} \\
& R_{2}(+) \rightarrow R_{3}, L_{4} \\
& R_{3}(-) \rightarrow L_{4} \\
& L_{4}(-) \rightarrow R_{1}, R_{2}, R_{3}
\end{aligned}
$$

For $\eta=(123)$, there are exactly two loops in $M(\theta)$ that fit the template for $\eta$; namely $W_{1}=R_{1} R_{2} L_{4}$ and $W_{2}=R_{2} R_{3} L_{4}$. So, $\theta$ forces $\eta$ and, in fact, $f_{\theta}$ contains exactly two distinct orbits of type $\eta$. Now of course, by Sarkovskii's theorem, for every $n, f_{\theta}$ must have a point of least period $n$. But it is evident from $M(\theta)$ that $\theta$ cannot force any cycle whose template contains 2 or more consecutive $L$ 's or 4 or more consecutive $R^{\prime} s$. In particular, $\theta$ cannot force $\eta=(136452)$ since the template for this $\eta$ begins with the RL pattern: $(R, R, L, R, L, L)$ which has 2 consecutive $L^{\prime}$ s. Further, $\theta=(1234)$ does force $\eta=(12435)$ as shown by the closed walk $W=R_{1} R_{2} L_{4} R_{3} L_{4}$. And, $\theta=(1234)$ forces $\eta=(135246)$ as seen by the closed walk $R_{1} R_{2} L_{4} R_{2} R_{3} L_{4}$. This last $\eta$ is a double of $\eta / 2=(123)$.

A cycle is called unimodal if there is exactly one turning point. For simplicity, let us assume a unimodal cycle has exactly one turning point and this turning point is a maximum. The forcing relation is a total order on the set of unimodal cycles [9]. The geometric perspective in this paper sheds light on the structure of this total order. For example, the template for a unimodal cycle cannot have 2 or more consecutive L's. And the structure of the oriented graph for a unimodal cycle is relatively uncomplicated. It is readily shown using the geometric algorithm described here that a unimodal cycle whose template contains $k$ consecutive $R$ 's must force any unimodal cycle whose template contains at most $j$ consecutive $R$ 's whenever $j<k$. 


\section{References}

[1] A. N. Sarkovskii, Coexistence of cycles of a continuous map of the line into itself, Ukr. Math. Z. 16 (1964) 61-71, in Russian.

[2] S. Baldwin, Generalizations of a theorem of Sarkovskii on orbits of continuous maps of the real line, Discrete Math. 67 (1987) 111-127.

[3] Alseda, LLibre, Misiurewicz, Combinatorial Dynamics and Entropy in Dimension One, World Scientific Publishing Co., (1993).

[4] I. Jungreis, Some results on the Sarkovskii partial ordering of permutations, Trans. Amer. Math. Soc. 325 (1991) 319-344.

[5] C. Bernhardt, E. Coven, M. Misiurewicz, I. Mulvey, Comparing Periodic orbits of Maps of the Interval, Trans. Amer. Math. Soc.333 (1992) no. 2 701-707.

[6] C. Bernhardt, E. Coven, A polynomial-time Algorithm for Deciding the Forcing Relation on Cyclic Permutations, Contemp. Math. 135 (1992) 85-93.

[7] C. Bernhardt, Simple Permutations with order a power of two, Ergodic Theory and Dynamical Systems 4 (1984), 179-186.

[8] L. Block, J. Guckenheimer, M. Misiurewicz, L-S Young, Periodic points and topological entropy of one-dimensional maps, Trans. Amer. Math. Soc. 300(1987) 297-306.

[9] P. Collet and J-P. Eckmann, Iterated Maps on the Interval as Dynamical Systems, Birkhauser, (1980). 